\title{
Recent LHCb results on CP violation in beauty decays to charmonia
}

\author{
Valeriia Lukashenko ${ }^{a, 1, *}$ \\ ${ }^{a}$ Nikhef, \\ Science Park 105, Amsterdam, the Netherlands \\ E-mail: valeriia.lukashenko@cern.ch
}

The latest three results from $\mathrm{LHCb}$ with decays of $\mathrm{B}$-mesons to charmonia states are presented. The first measurement of the CP-violating phase $\phi_{s}$ in the decay with electrons $B_{s}^{0} \rightarrow J / \psi\left(\rightarrow e^{+} e^{-}\right) \phi$ is shown. Results of an updated search of the rare decay $B^{0} \rightarrow J / \psi \phi$ are given. A precise measurement of the fragmentation fraction ratio of $B_{s}^{0}$ and $B^{0}, f_{s} / f_{d}$, extracted from a combination of most up-to-date $\mathrm{LHCb}$ results is reported.

*** The European Physical Society Conference on High Energy Physics (EPS-HEP2021), ***

*** 26-30 July $2021 * * *$

*** Online conference, jointly organized by Universität Hamburg and the research center DESY ***

${ }^{1}$ For the LHCb collaboration.

* Speaker 


\section{Introduction}

$\mathrm{CP}$ violation measurements are a possible key towards understanding the origin of matterantimatter asymmetry. Beyond Standard Model effects might enhance the CP-violation levels in comparison to the Standard Model predictions. Therefore, precise measurements of the Standard Model CP-violating parameters are important parts of both testing the Standard Model and looking for possible New Physics effects. The LHCb experiment is a forward detector, which was designed specifically for the forward boosted b-mesons, and is located at Large Hadron Collider (CERN) [1]. Unique capabilities of the detector in terms of the vertex reconstruction, particle identification and mass resolution give $\mathrm{LHCb}$ an advantage in terms of precise measurement of CP-violation.

The following results from the $\mathrm{LHCb}$ collaboration are presented in these proceedings: the first measurement of the CP-violating phase $\phi_{s}$ in the $B_{s}^{0} \rightarrow J / \psi\left(\rightarrow e^{+} e^{-}\right) \phi$ decays, search for rare $B^{0} \rightarrow J / \psi \phi$ decay, and precise measurement of the $f_{s} / f_{d}$ ratio of fragmentation fractions of B-mesons.

\section{First measurement of CP-violating phase in $B_{s}^{0} \rightarrow J / \psi\left(\rightarrow e^{+} e^{-}\right) \phi$ decays}

The CP-violating $\phi_{s}$ phase appears in the interference between mixing and direct $b \rightarrow c \bar{c} s$ transitions, shown in Fig. 1. The time-dependent CP-asymmetry $A_{C P}(t)$ in such decays is approximately proportional to the $\sin \left(\phi_{s}\right)$,

$$
A_{C P}(t) \propto \sin \left(\phi_{s}\right) \sin \left(\Delta m_{s} t\right)
$$

where $\Delta m_{s}$ is the mass difference between heavy and light mass eigenstates of $B_{s}^{0}$ meson. In the most general case, one can write the $\phi_{s}=-2 \beta_{s}$ phase as,

$$
\phi_{s}=\phi_{s}^{S M, \text { tree }}+\phi_{s}^{S M, \text { penguin }}+\Delta \phi_{s}^{N P}
$$

The penguin contribution $\phi_{S}^{S M, \text { penguin }}$ is expected to have a small contribution to the total $\phi_{S}$ phase [2]. Therefore, any significant deviation from the SM prediction would point to a source beyond the Standard Model.

In Fig. 1 the latest HFLAV combination of the $\phi_{s}$ and $\Delta \Gamma_{s}$ parameters are shown, where $\Delta \Gamma_{s}$ is the decay width difference between the heavy and light $B_{s}^{0}$ states [3]. Both parameters can be
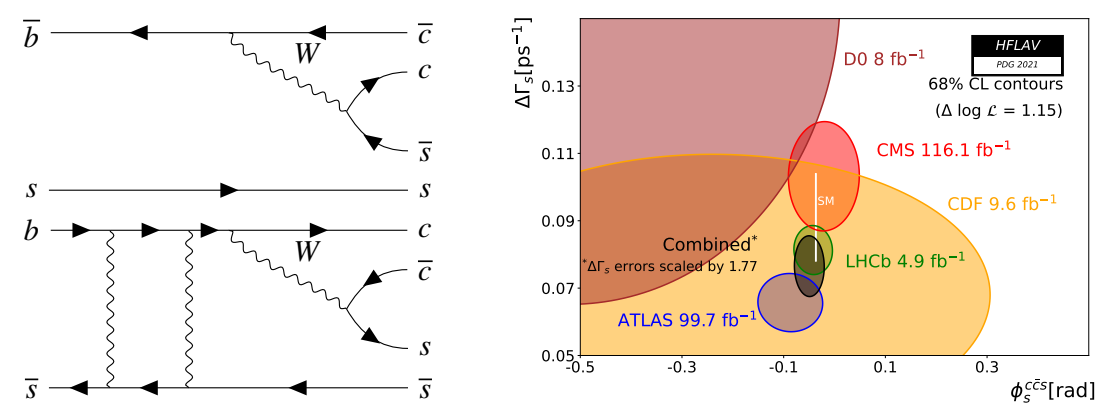

Figure 1: Direct and mixing Feynman diagrams of the $B_{s}^{0} \rightarrow J / \psi \phi$ decay and result of the HFLAV combination of different experimental results sensitive to both $\phi_{s}$ and $\Delta \Gamma_{s}$ from [3]. 
measured in the golden decay channel $B_{s}^{0} \rightarrow J / \psi \phi$, where $J / \psi \rightarrow \mu^{+} \mu^{-}$and $\phi \rightarrow K^{+} K^{-}$[4], [5]. As a complementary measurement one can use $B_{s}^{0} \rightarrow J / \psi\left(\rightarrow e^{+} e^{-}\right) \phi\left(\rightarrow K^{+} K^{-}\right)$decay, which is affected by different systematic uncertainty sources, but probes the same underlying physics effects as the golden decay channel [6]. In the following the $B_{s}^{0} \rightarrow J / \psi\left(\rightarrow e^{+} e^{-}\right) K^{+} K^{-}$will be referred as an electron mode and $B_{s}^{0} \rightarrow J / \psi\left(\rightarrow \mu^{+} \mu^{-}\right) K^{+} K^{-}$as a muon mode. The total data sample is $3 f b^{-1}$ collected by $\mathrm{LHCb}$ at $7 \mathrm{TeV}$ (2011) and $8 \mathrm{TeV}(2012)$. The electron mode statistics corresponds to about $10 \%$ of the muon mode statistics in this sample due to the imperfect reconstruction of electrons.

Since the $B_{s}^{0} \rightarrow J / \psi \phi$ is a pseudo-scalar particle to two vector-like particles decay, a nontrivial angular dependence of the final states has to be taken into account. It can be described by 3 polarization amplitudes: transverse parallel $A_{\|}$with angular momentum $L=2$, transverse perpendicular $A_{\perp}$ with $L=1$ and longitudinal $A_{0}$ with $L=0$. An additional amplitude $A_{S}$ coming from the $B_{s}^{0} \rightarrow J / \psi K^{+} K^{-}$decays without the intermediate $\phi$ resonance, is present in the measurement and is taken into account in the final time-dependent angular fit of the decay rate:

$$
\frac{d^{4} \Gamma\left(B_{s}^{0} \rightarrow J / \psi \phi\right)}{d t d \Omega}=\frac{1}{N} \sum_{k=1}^{10} f_{k}(\Omega) \varepsilon(t)\left(F T\left(B_{s}^{0}\right) \cdot h_{k}\left(t \mid B_{s}^{0}\right)+F T\left(\bar{B}_{s}^{0}\right) \cdot h_{k}\left(t \mid \bar{B}_{s}^{0}\right)\right) \otimes G\left(t \mid \sigma_{t}\right)
$$

where $\mathrm{N}$ is a normalization factor, that includes angular acceptance weights, $f_{k}(\Omega)$ are the angular functions, $\varepsilon(t)$ is the decay-time acceptance, $F T\left(B_{s}^{0}\right)$ is a correction from the imperfect flavor tagging algorithms, $h_{k}\left(t \mid B_{s}^{0}\right), h_{k}\left(t \mid \bar{B}_{s}^{0}\right)$ are time evolution functions and $G\left(t \mid \sigma_{t}\right)$ is decay time resolution.

Preselection of the signal candidates is done using kinematics, particle identification and Boosted Decision Tree cuts. The purity of the sample is further improved by applying the sWeight procedure [7]. The sWeights are computed from the fit to the $m\left(e^{+} e^{-} K^{+} K^{-}\right)$distribution in three categories depending on the Bremsstrahlung radiation recovery: no electrons with recovered Bremsstrahlung, one electron with recovered Bremsstrahlung and both electrons with recovered Bremsstrahlung. Time acceptance is estimated empirically from $B^{0} \rightarrow J / \psi K^{*}(892)$ data sample and is corrected via the Monte Carlo derived weights with respect to the signal decay. Angular acceptance is determined from the Monte Carlo simulation. Flavor taggers are calibrated using $B_{s}^{0} \rightarrow D_{s}^{-} \pi^{+}$sample. Decay time resolution is floated in the fit but constrained to the decay time resolution values retrieved from the prompt muon $B_{s}^{0} \rightarrow J / \psi K^{+} K^{-}$data sample, where the $J / \psi$ and kaons are not required to have a detached vertex from the interaction point of the incoming protons. Projections of the final time-dependent angular fit (3) are shown in Fig. 2. The CP-violating phase $\phi_{s}$ is measured to be $(0.00 \pm 0.28 \pm 0.05) \mathrm{rad}$ in the electron mode, which is consistent with the no CP-violation hypothesis. The biggest systematic sources for the electron mode come from the $m\left(e^{+} e^{-} K^{+} K^{-}\right)$mass model and decay time resolution constraint.

\section{Search for the rare decay $B^{0} \rightarrow J / \psi \phi$}

A non-resonant $B^{0} \rightarrow J / \psi\left(\rightarrow \mu^{+} \mu^{-}\right) K^{+} K^{-}$decay was observed in Ref. [8] with branching fraction of $(2.51 \pm 0.35 \pm 0.19) \times 10^{-6}$. A rare resonant decay $B^{0} \rightarrow J / \psi \phi$ contributes to the $B^{0} \rightarrow J / \psi K^{+} K^{-}$decay, but is suppressed by the Okubo-Zweig-Iizuka (OZI) rule [9]. According 

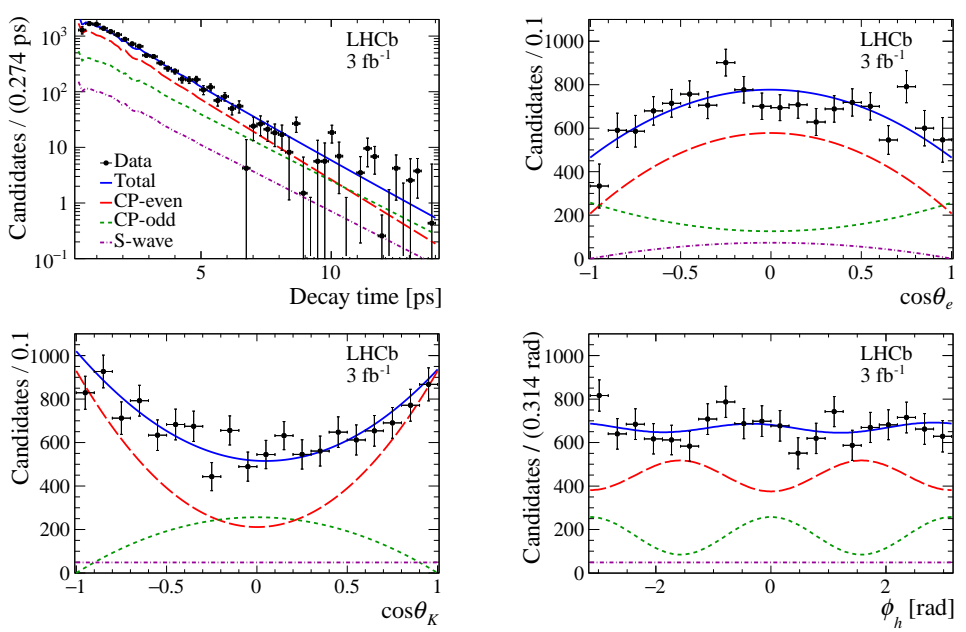

Figure 2: Results of the time-dependent angular fit projected to the four observables from [6]. The top left plot shows the projection onto the $B_{s}^{0}$ decay time, the top right plot onto the helicity angle between the electrons, the bottom left plot onto the helicity angles between kaons and the right bottom plot onto the angle between the decay planes of the kaons and the electrons. CP-even component includes both $A_{\|}$and $A_{0}$ amplitudes. CP-odd is $A_{\perp}$ amplitude. $A_{S}$ amplitude is called S-wave.

to the OZI rule, Feynman diagrams with disconnected quark lines are disfavored in comparison to other diagrams. The $B^{0} \rightarrow J / \psi \phi$ decay is an interesting probe for the OZI rule, which was previously excluded by $\mathrm{LHCb}$ at $\mathcal{B}\left(B^{0} \rightarrow J / \psi \phi\right)<1.9 \cdot 10^{-6}$ with $90 \% \mathrm{CL}$ using 2011 and 2012 data of $7 \mathrm{TeV}$ and $8 \mathrm{TeV}$ respectively [8]. Four production mechanisms of $\phi$ are possible in this decay: tri-gluon production, rescattering, photo-production and $\omega-\phi$ mixing, see Fig. 3. For an updated search a full LHCb dataset of $9 \mathrm{fb}^{-1}$, including $7 \mathrm{TeV}, 8 \mathrm{TeV}$ and $13 \mathrm{TeV}$ subsets, is used [9].

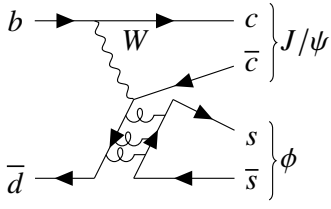

tri-gluon production

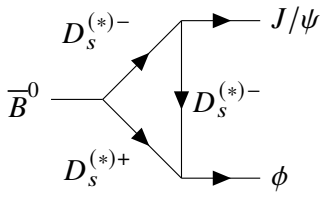

rescattering

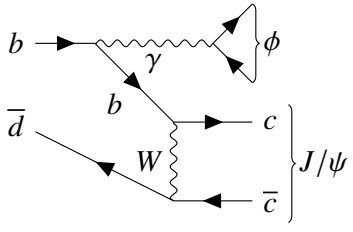

photo-production

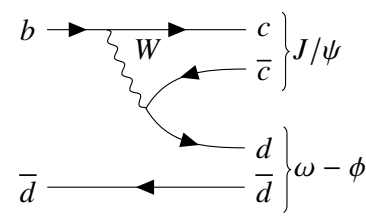

$\omega-\phi$ mixing

Figure 3: Four $\phi$ production mechanisms in the $B^{0} \rightarrow J / \psi \phi$ decay. The $\omega-\phi$ mixing has the highest expected branching fraction of $O\left(10^{-7}\right)[10]$.

The total number of $B^{0} \rightarrow J / \psi \phi$ events $\mathrm{N}$ is defined as,

$$
N=N_{\text {norm }} \times \frac{\mathcal{B}\left(B^{0} \rightarrow J / \psi\left(\rightarrow \mu^{+} \mu^{-}\right) \phi\right)}{\mathcal{B}\left(B_{s}^{0} \rightarrow J / \psi\left(\rightarrow \mu^{+} \mu^{-}\right) \phi\right)} \times \frac{\varepsilon_{B^{0}}}{\varepsilon_{B_{s}^{0}}} \times \frac{1}{f_{s} / f_{d}}
$$

where $N_{\text {norm }}$ is the yield of the normalization decay channel $B_{s}^{0} \rightarrow J / \psi \phi, \mathcal{B}$ are the corresponding branching fractions, $\varepsilon$ are efficiencies and $f_{s}, f_{d}$ are $B_{s}^{0}$ and $B^{0}$ fragmentation fractions. To preselect signal candidates, first the peaking background from $\Lambda_{b}^{0} \rightarrow J / \psi p K^{-}$is vetoed with kinematics cuts, after which simulation-trained Boosted Decision Tree cuts are applied together with kinematics and 
particle identification cuts. A sequential mass fit is used to estimate the signal yield. The sequential mass fit procedure starts with first fitting $m\left(J / \psi K^{+} K^{-}\right)$for both the signal and normalization decays. In the second step the yields of $\Lambda_{b}^{0} \rightarrow J / \psi p K^{-}$events that remain in the data sample, combinatorial background and non-resonant decays extracted from the $m\left(J / \psi K^{+} K^{-}\right)$fit are used to fix the background yields in the $m\left(K^{+} K^{-}\right)$fit around the $\phi$ mass. This is done separately in the signal mass window with $m\left(J / \psi K^{+} K^{-}\right)$around the $B^{0}$ mass and normalization mass window with $m\left(J / \psi K^{+} K^{-}\right)$around the $B_{s}^{0}$ mass. Results of the sequential mass fit procedure are shown in Fig. 4. No significant signal yield is observed, and an upper limit on the branching fraction is set at the $90 \%$ CL to be $1.1 \cdot 10^{-7}$.

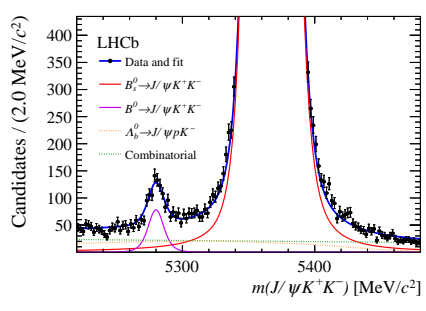

$m\left(J / \psi K^{+} K^{-}\right)$fit

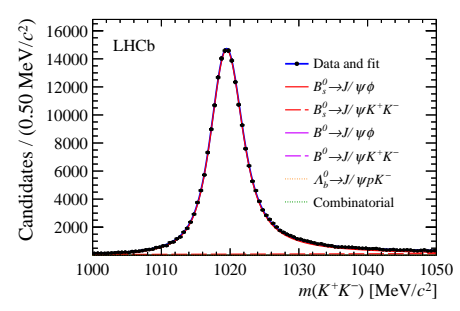

$m\left(K^{+} K^{-}\right)$normalization fit

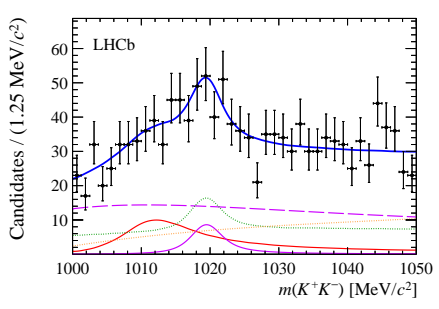

$m\left(K^{+} K^{-}\right)$signal fit

Figure 4: Results of the sequential mass fit procedure for both signal $B^{0} \rightarrow J / \psi \phi$ and normalization $B_{s}^{0} \rightarrow J / \psi \phi$ decays from [8]. Only 2015-2018, $13 \mathrm{TeV}$ dataset of about $6 \mathrm{fb}^{-1}$ is shown.

\section{Precise measurement of the $f_{s} / f_{d}$ ratio of fragmentation fractions}

The fragmentation fraction is a probability of a $\mathrm{b}$ quark to hadronize into one of the $\mathrm{B}$ hadrons. The ratio of the fragmentation fractions $f_{s} / f_{d}$ is a major source of systematic uncertainty for most $B_{s}^{0}$ meson branching fraction measurements [11]. It has been shown that $f_{s} / f_{d}$ depends on $p_{T}$ and might have a non-negligible dependence on $\sqrt{s}$. In order to extract the most precise up-to-date value of $f_{s} / f_{d}$, multiple LHCb results are combined. The LHCb measurements that determine the ratio of efficiency-corrected yields of $B_{s}^{0}$ to $B^{+}$or $B^{0}$ can be divided into three main groups: those using semileptonic final states, those using hadronic final states and those using charmonia final states. Semileptonic final states included are $B_{s}^{0} \rightarrow D_{s}^{-} X \mu^{+} v_{\mu}, B_{s}^{0} \rightarrow \bar{D} \bar{K} X \mu^{+} v_{\mu}, B^{+, 0} \rightarrow \bar{D}^{0} X \mu^{+} v_{\mu}$ and $B^{+, 0} \rightarrow D^{-} X \mu^{+} v_{\mu}$. Hadronic decay modes included are $B^{0} \rightarrow D^{-} \pi^{+}, B^{0} \rightarrow D^{-} K^{+}$and $B_{s}^{0} \rightarrow D_{s}^{-} \pi^{+}$. Charmonia decay modes are $B_{s}^{0} \rightarrow J / \psi \phi, B^{+} \rightarrow J / \psi K^{+}$. In Fig. 5 one can find results of the fits to the $p_{T}$ dependence of $f_{s} / f_{d}$ from different measurements. The extracted integrated values of $f_{s} / f_{d}$ at the three center-of-mass energies with $p_{T} \in[0.5,40] \mathrm{GeV} / \mathrm{c}$ and $\eta \in[2 ., 6.4]$ are found to be: $f_{s} / f_{d}(7 \mathrm{TeV})=0.2390 \pm 0.0076, f_{s} / f_{d}(8 \mathrm{TeV})=0.2385 \pm 0.0075$, $f_{s} / f_{d}(13 \mathrm{TeV})=0.2539 \pm 0.0079$. Using the updated $f_{s} / f_{d}$ value, as well as updated values of the input normalization channel branching fractions, the previous $B_{s}^{0}$ branching fraction measurements are updated. Using $\mathcal{F}_{R}$, the ratio of the branching fractions of $B_{s}^{0} \rightarrow J / \psi \phi$ and $B^{+} \rightarrow J / \psi K^{+}$, $\mathcal{B}\left(B_{s}^{0} \rightarrow J / \psi \phi\right)$ is measured to be $(1.018 \pm 0.032 \pm 0.037) \cdot 10^{-3} \cdot \mathcal{B}\left(B_{s}^{0} \rightarrow D_{s}^{-} \pi^{+}\right)$, determined using data driven inputs instead of the theory inputs, is found to be $(3.20 \pm 0.10 \pm 0.16) \cdot 10^{-3}$. 

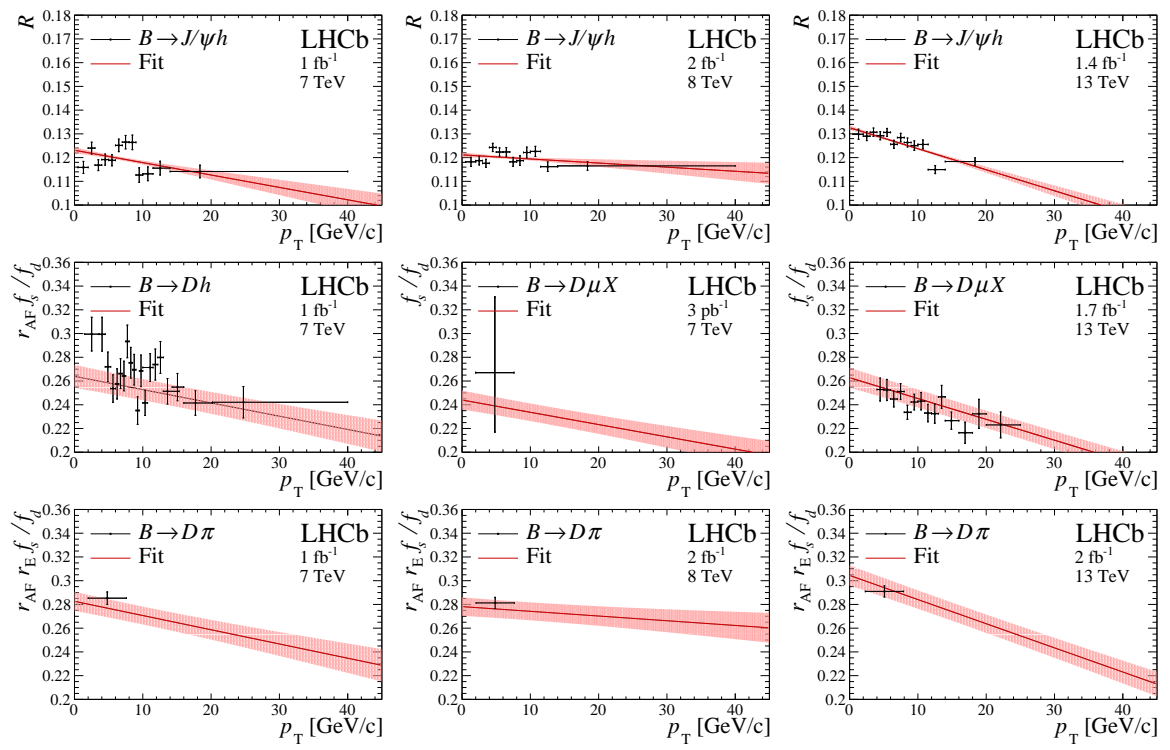

Figure 5: $f_{s} / f_{d}$ as a function of the B-meson $p_{T}$ for different measurement sets from [11]. R is the efficiency corrected yield given by $R=\mathcal{F}_{\mathcal{R}} \cdot f_{s} / f_{d}$, where $\mathcal{F}_{\mathcal{R}}$ is defined in the text. $r_{A F}$ and $r_{E}$ are scale factors related to the theoretical inputs to hadronic and exchange diagrams. The fit model is defined per center-of-mass energy as $f_{s} / f_{d}\left(p_{T}, \sqrt{s}\right)=a_{i}+b_{i} \cdot p_{T}$ where $\mathrm{i}=7 \mathrm{TeV}, 8 \mathrm{TeV}, 13 \mathrm{TeV}$.

\section{References}

[1] A. Alves and et al. Journal of Instrumentation 3 (2008) S08005.

[2] R. Aaij and et al. Physics Letters B 742 (2015) 38-49.

[3] Y. Amhis and et al. The European Physical Journal C 81 (2021) 226.

[4] R. Aaij and et al. Physical Review Letters 114 (2015).

[5] R. Aaij and et al. The European Physical Journal C 79 (2019) .

[6] R. Aaij and et al. 2105.14738.

[7] M. Pivk and F. Le Diberder Nucl. Instrum. Methods Phys. Res. A 555 (2005) 356-369.

[8] R. Aaij and et al. Physical Review D 88 (2013).

[9] R. Aaij and et al. Chinese Physics C 45 (2021) 043001.

[10] M. Gronau and J.L. Rosner Physics Letters B 666 (2008) 185-188.

[11] R. Aaij and et al. Physical Review D 104 (2021). 